\title{
MUlTi-CRITERIA HANDOVER DECISION FOR HETEROGENEOUS NETWORKS: CARRIER Aggregation DePloyment SCENARIO
}

\author{
Maryam Abdulazeez- Ahmed, Nor Kamariah Nordin, Aduwati Bint Sali and \\ Fazirulhisyam Hashim
}

Department of Computer and Communication Systems Engineering, Universiti Putra Malaysia, 43400, UPM Serdang, Selangor Malaysia

\begin{abstract}
Carrier aggregation and integration of heterogeneous networks allow the mobile user equipment to benefit from wider bandwidth and radio coverage of different access technologies. However, these technologies have increased handoff scenario probability through user equipment's mobility, leading to high outage probability and low throughput. Handover is an important aspect of mobility management which allows users to migrate from one cell to another without losing connections. But no lone access technology meets the requirements of providing seamless communication without loss and delay. Therefore, high-quality service continuity and reliable user equipment access to network anywhere and at any time require the design of an appropriate handover decision algorithm. In this paper, a multi-criteria based handover decision-making algorithm is proposed to evade loss of communication and provide better performance to the system. It adaptively makes handover decisions based on different decision criteria (load, availability of resources, and the handover scenario type) in addition to signal interference to noise ratio. The multicriteria handover decision making algorithm-based method chooses among the cells that satisfy the requirements for the handover. As compared to some existing handover decision algorithms, simulation result shows that this algorithm improves system performance in terms of handover failure by $93 \%, 72 \%$, and $58 \%$; radio link failure reduction by $77 \%, 43 \%$, and $22 \%$; and handover ping-pong by $81 \%$, 59\%, and $36 \%$ over the conventional received signal strength, received wireless transmission line, and multiinfluence factor hand over decisions respectively.
\end{abstract}

\section{KEYWORDS}

Carrier aggregation, handover decision, user equipment, heterogeneous network, Long Time EvolutionAdvanced, multi-criteria, signal-to-interference-plus-noise-ratio, interference-to-interference-plus-noiseratio

\section{INTRODUCTION}

Several works have proposed various handover decision algorithms in wireless communication systems. In conventional handover, Received Signal Strength (RSS) and/or power level received from candidate base stations is compared in [1]. Also, other parameters such as: (a) RSS with a threshold (b) RSS with hysteresis (c) RSS with hysteresis and threshold [2][3] (d) RSS with hysteresis and distance [4][5] (e) Signal-to-Interference-plus-Noise-Ratio (SINR) [6][7][8][9] and (f) Interference-to-Interference-plus-Noise-Ratio (IINR) [10]. However, their handover decision algorithms are based on a single parameter. In order to enhance handover decision through user's movement, other factors like bandwidth and availability of resources, velocity of mobile terminal, distance between mobile stations, and Evolved Node Bs (eNodeBs) need to be considered so as to maximize system performance and User Equipment (UE) satisfaction. This is because 
traditionally, mobile devices may be moved for better service quality. Thus, employing only single criteria causes service interruption and unbalanced network load which in turn degrades user's throughput and increases its outage probability.

In [11][12][13][14], handover decisions are taken based on multi-criteria factors but none considers carrier aggregation techniques. Carrier aggregation in heterogeneous networks leads to increased access in technologies, bandwidth, transmission rate, energy requirement, coverage, and enhanced network protocols. For the purpose of efficiency, one infrastructure capable of interconnecting multiple access networks is required to support data concurrence at all cell locations. Thus, efficient handover mechanism is required for ensuring seamless connectivity and uninterrupted service delivery for future generation wireless networks.

Carrier Aggregation (CA) is one of the main background features for Long Time EvolutionAdvanced (LTE-A). With CA, users would gain access to a total bandwidth of up to $100 \mathrm{MHz}$. The system bandwidth may be contiguous, non-contiguous, and inter-band noncontiguous [15]. The bandwidth of these Component Carriers (CCs) can vary widely, ranging from $1.4 \mathrm{MHz}$ to $20 \mathrm{MHz}$ for Long Time Evolution (LTE) carriers. Also, propagation characteristics differ in different CCs. For example, the propagation characteristic of CCs in the $800 \mathrm{MHz}$ band differs from a component carrier in the $2.4 \mathrm{GHz}$ band [16].

Also, the introduction of CA technology, brings with it, a new handover scenario, which can be performed between the $\mathrm{CCs}$ serving under the same sector and the same eNodeB with a probability of redefining the Primary Component Carriers (PCCs). This results in increased handover probability that consequently increases throughput degradation. The handover scenario here is being translated into an efficient Handover Decision (HoD) provided the serving PCC gives enough RSS to the UEs on acceptable levels.

Though [17] has studied handover decisions in the CA scenario using multi-influence criteria, but only macro celle NodeB is considered. This cannot give the maximum benefit of CA in terms of capacity and coverage increment. On the other hand, a limited number of UEs can be served by outdoor macro eNodeB in heavily populated areas with the available spectrum. Therefore, the CA technique and heterogeneous networks are possible solutions [18][19][20] to improve cell capacity and utilization of the available spectrum. On the other hand, the seamless mobility of UEs in the CA scenario using the same parameters of a macro eNodeB faces challenges due to the aggregation of multiple CCs [21].

Fundamentally, handover is considered during the period when a voice call is in progress and moving toward the current eNodeB (i.e source to a target eNodeB). In this case, signal measurements obtained at a UE from the neighboring eNodeB, are reported by the UE to the serving eNodeB and the handover decision is based on the measurement report from the UE at the source eNodeB. Whereas in CA-based system, due to multiple CCs, the source and the target eNodeBs can be CCs of the same or different eNodeBs. Employing such a signal Measurement Report (MR) for decision making is not speedily concluded. Thus, it leads to a high probability of handover failure, increase throughput degradation, and user outage probability especially to the high-velocity UEs [22][23].

The main idea of handover is to maintain the connection while the subcarrier moves out of the coverage area of the source eNodeB and enters the vicinity of the target eNodeB. Availability of services to satisfy the customer is one of the major goals in cellular networks communication [24]. However, efficient handover decision algorithm leads to an improvement in continuous connectivity and enhancement in the network performances are inadequate. Therefore, it is novel 
International Journal of Computer Networks \& Communications (IJCNC) Vol.12, No.4, July 2020

to look into $\mathrm{HoD}$ algorithm that can satisfactorily handle the major tasks in cellular network by enhancing the level of the services, and manage the resources of the network simultaneously, and serve as motivation to carrying out this work.

In this work, a proposed algorithm called Multi-criteria Handover Decision (MCHoD) is presented to take tactful decision on handover when heterogeneous Network (HetNet) and CA is implemented in LTE-A system. MCHoD dynamically changes handover decision algorithm based on the signal strength, availability of resources, UE distance from the serving eNodeB and the handover scenario type. MCHoD algorithm seeks to improve the system performances by providing higher efficiency in SINR, cell edge spectral efficiency, and low outage probability in the event of users' mobility. The major contribution of this work is the development of MCHoD algorithm on CA, integrating macro-eNodeBs (MeNodeBs) and femto-eNodeBs (FeNodeBs) to provide UEs uninterrupted services in users' mobility network.

The remaining of this paper is organized as follows. Related Work is presented in Section 2 followed by handover with CA techniques in section 3 . The proposed algorithm and the system model are described in Section 4. In section 5, results and discussions are presented while conclusion is given in Section 6.

\section{RELATED WORK}

The handover decision is taken by the serving eNodeB based on the measurement report that is received from the served UE. MR contains a list of signal levels of neighboring cells, and it can provide other information based on the HoD algorithm being implemented. There are several methods of a handover decision algorithm. Generally, it may be classified into the following categories:

1) The traditional RSS-based criteria

2) Fuzzy logic

3) Multi-influence Factor (MIF) based

RSS-based approach is the traditional handover decision algorithm that uses RSS as the main criteria to trigger handover [2][25]. In this case, the signal strengths of neighboring eNodeBs are compared and consequently, the handover is enabled. The RSS-based technique has been enhanced by adopting the RSS threshold (hysteresis) [5] and by combining the RSS threshold with the user's distance [4], SINR [6], and IINR [10]. To suppress the Ping-Pong effects, a threshold is defined and applied in relative signal strength. The handover is initiated if existing eNodeB RSS is lower than the threshold value and the new eNodeB RSS is stronger than the threshold. Though, RSS based method is simple in principle and easy to use in measuring the characteristics and the quality of services. However, the RSS signal fluctuates due to path loss in the wireless medium. This effect results in unnecessary handovers, ping pong, and throughput degradation. Also, RSS with threshold has drawbacks of handover delay and high call drop.

However, recent studies have demonstrated that by implementing CA and HetNets technologies, handover decision becomes more challenging. The RSS-based criterion is not complete enough for a decision process in HetNet with CA technique.

There are studies which employed fuzzy logic in decision making process to improve the intelligence of HoD [26][27] for network selection and performances. Fuzzy logic method has advantage of inherent potential to solve the precision problems but with the cost of design complexity. 
Meanwhile, an MIF decision making criteria was studied in a coordinated carrier aggregation deployment scenario [17]. MIF applied different parameters that influence the decision making process. Though, the simulation result shows that ping pong has been reduced but the result is not optimal when HetNet is considered in CA.

The algorithm based on multi-criteria reduces the handoff delay and service disruption time. This in turn decreases the packet lost, and increases throughput during handover [11][13][28]. Because of this, in MCHoD the chosen criteria ensure that the accuracy of the decision making is held high. The main criteria are either user-related or network related, such as RSS, mobility, application, and bandwidth. Despite all the earlier works are based on different criteria in HetNets and CA, the question of which of the parameters yield an optimal handover process is an open question. So, to find out the best parameters for successful handover process, a new algorithm called multi-criteria handover decision algorithm is proposed for efficient handover decision making when CA and HetNet is considered in LTE-A system. Table 1 compares some of the existing work on different algorithms.

Table 1. Comparison of related works

\begin{tabular}{|l|l|l|l|}
\hline \multicolumn{1}{|c|}{ Algorithm } & Application & Achievement & \multicolumn{1}{c|}{ Drawback } \\
\hline RSS & HetNet & Simple & $\begin{array}{l}\text { Inaccurate } \\
\text { High rate of unnecessary } \\
\text { handover } \\
\text { Ping-pong effect }\end{array}$ \\
\hline Fuzzy Logic & HetNet & Accurate precision & High design complexity \\
\hline MIF & Coordinated CA & $\begin{array}{l}\text { Ping pong effect and } \\
\text { unnecessary } \\
\text { handover reduced }\end{array}$ & Only single eNodeB was used \\
\hline
\end{tabular}

Therefore, the proposed MCHoD algorithm combines RSS, bandwidth, speed, handover scenario, and SINR in decision process in other to achieve seamless mobility.

\section{Carrier Aggregation based Handover Techniques in Hetnets}

The introduction of CA technique in LTE-A system raises the number of aggregated CCs that can be deployed at one eNodeB and simultaneously assign it to a UE. These CCs are grouped into two main types. The first one is known as a PCC, whereas the second type of CCs is called Secondary Component Carrier (SCC) [29][30].

The CCs concept of CA techniques considered in this study are as follows. We assumed that the PCC is always at the Micro eNodeB (MeNodeB), whereas, the SCC is at the Femto eNodeB (FeNodeB). With this configuration, the UEs always have a stable anchor PCC. At the commencement of radio resource connection (RRC), UE is associated to the PCC which provides the best signal quality over all the active CCs as the serving cell that is always active throughout the active mode of the UE [31][32][33]. Data transmission between the UE and the eNodeB; and the control signaling information exchange are usually carried out with PCC. Apart from being used as data transmission channel, PCC is employed for random access procedure and allocation of SCC. In the process, radio link failure event is recorded only if there is radio link connection failure over the PCC. On the other hand, SCC can be configured and activated by eNodeB as an additional $\mathrm{CC}$ used for additional resources for the served UE where higher data rate is required. 
However, SCC cannot be used for exchanging control signaling messages between a UE and an eNodeB [34].

Addition more to the developments, Radio Link Failure (RLF) is an indicator recorded when a connection over the PCC fails. If this happens, Radio Resource Control (RRC) re-establishment procedure comes into action over the named PCC. In the same vein, the Non-access Stratum $(\mathrm{NaS})$ recovery procedure is triggered if the RCC re-establishment procedure over the PCC is failed within T310. T310 is the maximum allowed time for recovering connection through the RRC re-establishment procedure [30][35].

Unlike the LTE system, the CA in the LTE-A system has increased handover scenarios (i.e. changing of $\mathrm{PCC}$ in the same sector) influenced by aggregation of multiple carriers in addition to channel conditions of two or more adjacent cells that may completely differ for the specific UE. This poses challenges for the target eNodeB to guarantee the reservation of sufficient system resources for the incoming UE's service requirement. Therefore, employing single criteria in the handover decision-making process may result in inappropriate handover for the target eNodeB thus, leading to high handover probability which in turn increases outage probability and throughput degradation.

In this paper, in order to reduce the outage probability and increase the spectral efficiency, we propose a multi-criteria handover decision method with additional parameters such as SINR, bandwidth, and data rate. This algorithm provides a set of parameters for better handover.

\section{Proposed Multi-Criteria Handover Decision Algorithm}

In response to handover decision challenges of CA concept in LTE-A and future generation systems, different handover decision algorithms have been proposed but none of the algorithms has given the optimal result expected, particularly for HetNet's Carrier Aggregation Deployment Scenario (CADS). Therefore, in this study, we propose an efficient multi-criteria handover decision (MCHoD) algorithm that utilizes both the network parameter (network topology and radio resources) and mobile parameter (load, and UE distance) as input for decision making.

Due to the deployment structure of Het-Net's CADS, UEs may not receive a signal from only the MeNodeB, but also from FeNodeB. UEs are mostly connected to MeNodeB for high signal reception and quality of service but are handed over to FeNodeB and vice-versa when the signal strength drops below acceptable levels. This consequently leads to load imbalance. Furthermore, differences in the transmitted power between MeNodeB and FeNodeB often cause frequent handover, especially, ping-pong handover which negatively affects the system throughput. Therefore, in this study, we incorporate Cell Range Expansion (CRE) power control technique standardized in the Third Generation Partnership Project (3GPP) [36], as a virtual bias, added to actual UE received power. This assists the UE with the association decision required. CRE is a practical power control technique that boosts the received signal power strength of FeNodeB which forces the UEs to offload to it. The biasing technique limits the MeNodeB load and stabilizes the UE when it transits around the cell edges. At the same time, it reduces ping-pong probability and consequently increases the network throughput.

Meanwhile, CRE will also alter the UE's handover position due to changes in the coverage area by the FeNodeB. During the UE association process, the handover margin is used to adapt the received signal strength (RSS) in MCHoD. The PCC handover decision is initiated by UE performance parameters such as SINR Handover management (HoM) and SINR threshold; and 
International Journal of Computer Networks \& Communications (IJCNC) Vol.12, No.4, July 2020

handover scenario types. With this, handover decisions can be adaptively executed according to the following conditions as depicted in the flowchart in Figure 1.

1) If the target cell SINR is larger than the sum of the handover margin and the serving cell SINR then handover to target cell for all UEs on PCC, subject to condition 2.

2) That the target cell load is less than the sum of the serving cell load and the load margin on inter-frequency components.

On the other hand, if the handover type is changing the serving sector or serving eNodeB (macro to Femto), the decision will be based on resource availability, UE distance to the eNodeB, and SINR. The handover decision can then be executed if the following conditions are satisfied:

1) That the target SINR is larger than that of the serving SINR plus handover margin

2) That the serving cell load is larger than the target cell load by a predefined load margin

3) That the UE distance to the target cell is less than the UE distance to the serving cell.

The combination of both the MCHoD and CRE techniques does not only increase the system throughput but also reduces the handover failure rate.



Figure 1: Flow diagram of the proposed multi-criteria decision algorithm 
International Journal of Computer Networks \& Communications (IJCNC) Vol.12, No.4, July 2020

\subsection{System Model and Simulation Setup}

The proposed algorithm is implemented as a multicell system using MATLAB toolbox systemlevel simulator to generate LTE-A heterogeneous network topologies. MATLAB have a wide variety of inbuilt libraries and features that can convert $\mathrm{C}, \mathrm{C}++$ code into MATLAB codes. It also allows the necessary manipulation of data, improves the chances of new technology, and increases the security surrounding a new technology [33]. We employed MATLAB as used by prior studies [17][11][1].

The network scenario configuration was implemented by overlaying FeNodeBs on the MeNodeBs coverage area with a uniform user's distribution using the 3GPP LTE-A standard topology [34][37]. The considered simulation environment consists of 61 hexagonal cell layout of macro eNodeB located at the center with 500m inter-site distance. The MeNodeBs is subdivided into three sectors and Omni-directional coverage femtocells are deployed in each sector to form the CCs in inter-band noncontiguous deployment. The operating frequencies for CC1 and CC2 are $2 \mathrm{GHz}$ and $3.5 \mathrm{GHz}$ respectively premised on the band scenarios for Release 13 and 14 [34]. The UEs are uniformly distributed across the femtocell coverage area. The simulated cell accommodates up to 50 mobile UEs randomly moving within the coverage area with a uniform speed of $3,20,30,60,90$, and $120 \mathrm{~km} / \mathrm{h}$. The assumption is that the frequency re-use factor is taken to be 1 . The inter-cell interference to other sectors is generated only from the first tier of the neighboring eNodeBs. This is because the second tier neighboring eNodeBs are far away from the serving eNodeB. Moreover, their signals are assumed to be weak and therefore, have no significant impact on the served UEs. Radio resource control (RRC), radio link failure (RLF), and $\mathrm{NaS}$ recovery procedures are all considered in this simulation. Adaptive Modulation and Coding (AMC) schemes as specified in [38] is considered. The handover process follows the procedures introduced in [39] with the assumption of $600 \mathrm{~ms}$ as a time to trigger and $6 \mathrm{~dB}$ as handover margin. In order to improve performance accuracy; Radio Link Failure (RLF), radio resource connection re-establishment, and NaS procedures are considered throughout the simulation. The summary of the parameters that are used is listed in Table 2.

Table 2. Simulation parameters and values

\begin{tabular}{|l|l|}
\hline Parameters & Values \\
\hline Propagation model & Macrocell/Femtocell urban model \\
\hline \multicolumn{2}{|c|}{ OFDM } \\
\hline Number of Component Carriers & $2 \mathrm{CCs}$ each $20 \mathrm{MHz}$ \\
\hline Bandwidth (MHz) & $40(2 \mathrm{X} 20) \mathrm{MHz}$ \\
\hline Number of Resource Block & 100 \\
\hline Resource Block size & $180 \mathrm{kHz}$ \\
\hline Number of Subcarrier per RB & $12 \mathrm{Subcarrier}$ per RB \\
\hline Subcarrier Spacing & $15 \mathrm{kHz}$ \\
\hline Number of OFDM Symbol per frame & 7 \\
\hline \multicolumn{2}{|c|}{ MeNodeB Parameters } \\
\hline Transmit power (dBm) & $46 \mathrm{dBm}$ \\
\hline Antenna gain + feeder loss & $15 \mathrm{dBi}$ \\
\hline Antenna height & $15 \mathrm{~m}$ \\
\hline Noise figure & 5 \\
\hline \multicolumn{2}{|c|}{ FeNodeB Parameters } \\
\hline Transmit power (dBm) & $10 \mathrm{~dB}$ \\
\hline Antenna gain + feeder loss & $2 \mathrm{dBi}$ \\
\hline
\end{tabular}


International Journal of Computer Networks \& Communications (IJCNC) Vol.12, No.4, July 2020

\begin{tabular}{|l|l|}
\hline Antenna height & $1 \mathrm{~m}$ \\
\hline Noise figure & $4 \mathrm{~dB}$ \\
\hline Biasing value & 10 \\
\multicolumn{2}{|c|}{ User Parameters: } \\
\hline \multicolumn{2}{|c|}{} \\
\hline Antenna Gain & $0 \mathrm{dBi}$ \\
\hline Antenna Height & $1.5 \mathrm{~m}$ \\
\hline Noise Figure & $9 \mathrm{~dB}$ \\
\hline Thermal Noise Power (NP) & $\mathrm{NP}=\mathrm{Nt}+10 \log (\mathrm{BW} \times 106) \mathrm{dB}$ \\
\hline Time-To-Trigger (TTT) & $600 \mathrm{~ms}$ \\
\hline Handover Margin (HoM) & $6 \mathrm{~dB}$ \\
\hline T311 & $10 \mathrm{~s}$ \\
\hline Measurement Interval for PCC and SCC & $50 \mathrm{~ms}$ \\
\hline Process Delay Time & $10 \mathrm{~ms}$ \\
\hline Simulation Time & $500 \mathrm{~min}$ \\
\hline
\end{tabular}

\section{RESUlt AND DisCuSSION}

The proposed MCHoD algorithm is simulated and validated using MATLAB tools for the simulation following the evaluation methodology of the LTE-A system.

Generally, high-speed UEs present several challenges to handover algorithms. To evaluate the performance of $\mathrm{MCHoD}$, it is compared with other handover decision algorithms such as Conventional Received Signal Strength (CRSS), Received Wireless Transmission Line (RWTL), and Multi-influence Factor (MIF) earlier discussed in section II. To demonstrate the effectiveness of our algorithm, a comprehensive investigation of $\mathrm{MCHoD}$ at various speeds was examined based on the probabilities of the Radio Link Failure (RLF), Handover Failure Rate (HFR), pingpong and throughput as shown in Figures 2, 3, and 4 respectively.

In terms of Handover Failure (HoF) reduction, the performance gains of MCHoD over CRSS, MIF-HoD, and RWTL is presented in Figure 2. Generally, it is observed that at low speeds, the rate of HoF is lower than at high speeds as in Figure 2a. This can be attributed to the fact that the number of cells that a UE crosses over a certain period of time is lower at those speeds (i.e. 3, 20, and $30 \mathrm{~km} / \mathrm{h}$ ). However, an increase in UE's speed accelerates the frequency of handover because as the UE moves away from the serving cell, signal reception becomes weaker, and so also is the data rate. This results in a high $\mathrm{HoF}$ rate and consequently reduces the performance of the system.

A plot of the average HoF probability shows that CRSS has the highest HoF rate as shown in Figure $2 \mathrm{~b}$. The reason is that CRSS employs only a single criterion for handover decision making. Besides, it does not consider the SINR and the availability of the resources. Therefore, it suffices to say that, since handover consumes more resources, it is bound to experience more $\mathrm{HoF}$ rates if the target cell is not having the required resources. The other two baselines, RWTL and MIF have a much lower HoF compared to CRSS because the former utilizes RSS and transmission line losses whereas, the latter employs more than one criterion in the handover decision making process implementing CA scenario. The use of CA facilitates wider bandwidth and effective utilization of available resources to make decisions. Quantitative assessment produced shows that MCHoD achieves higher reduction gain in HoF by approximately $93 \%$, $72 \%$, and 58\% over CRSS, RWTL-HoD, and MIF-HoD respectively. 
International Journal of Computer Networks \& Communications (IJCNC) Vol.12, No.4, July 2020

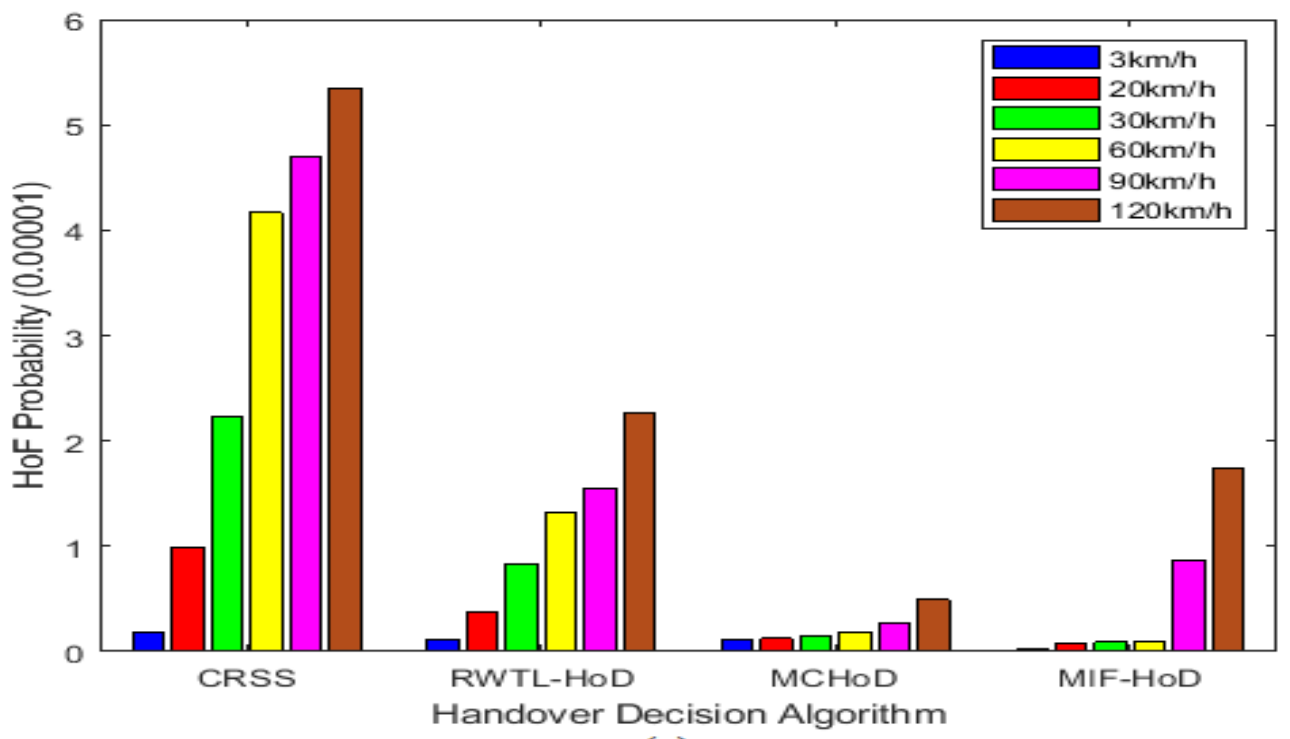

(a)

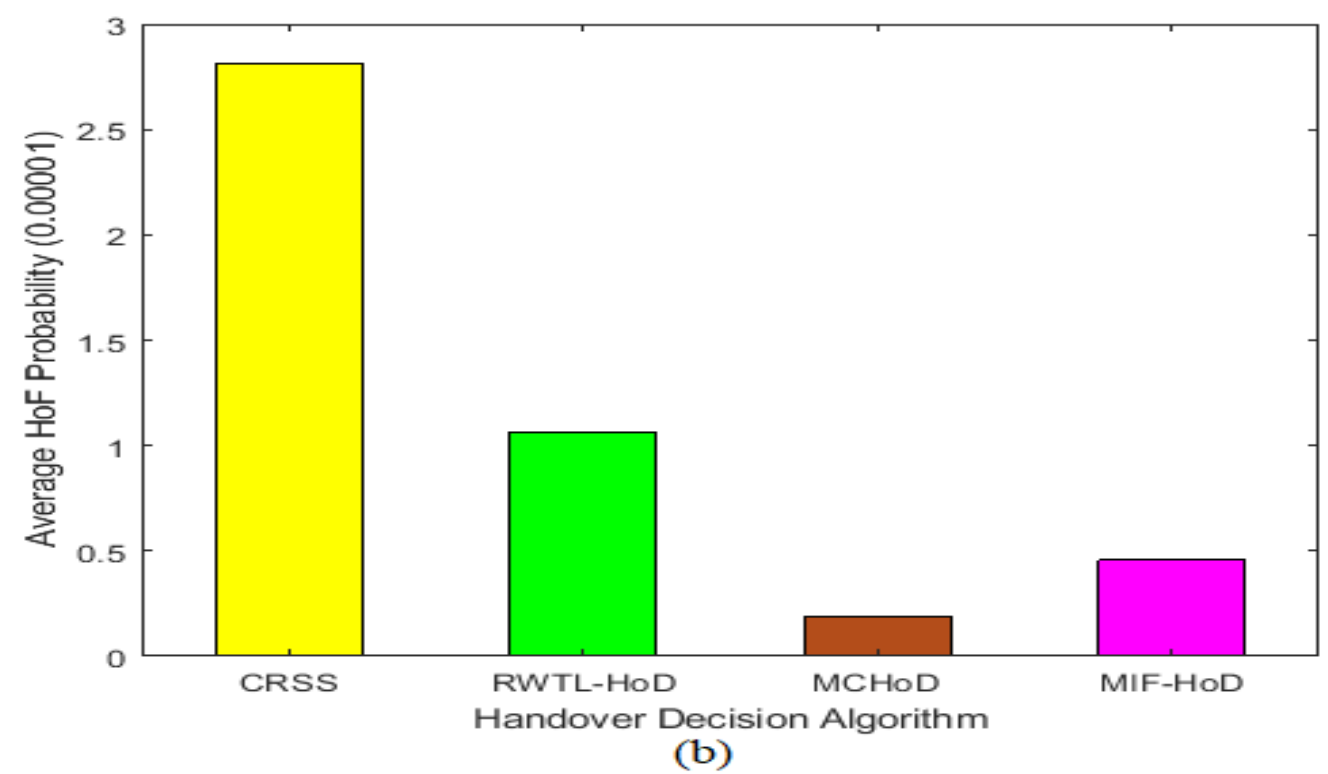

Figure 2: Probability of handover failure (HoF). (a) At different speeds (b) average handover failure probability for different decision algorithms.

UE speed does not only affect handover, it has much to do with RLF probability. At high UE speeds, radio link connection becomes weakened due to fading, leading to higher RLF failure. Figure 3a illustrates the RLF rate at different speeds. Overall, it is observed that the probabilities of RLF get higher as the UE's speed increases but the effects vary among the four algorithms examined. Similar to the evaluation of HoF, CRSS is much affected by RLF than the other algorithms. Generally, RSS is known to be unstable [26]. So, its implementation as an only deciding factor in the heterogeneous network which has different characteristic cannot give the optimal result. Figure $3 \mathrm{a}$ shows the performances of RFL in relation to mobile speed where MCHoD and MIF-HoD are both low. On the average (Figure 3b), MCHoD shows a good performance (low RLF ratio) compared with the other algorithms, CRSS, RWTL, and MIF-HoD by $77 \%, 43 \%$, and $22 \%$, respectively. 

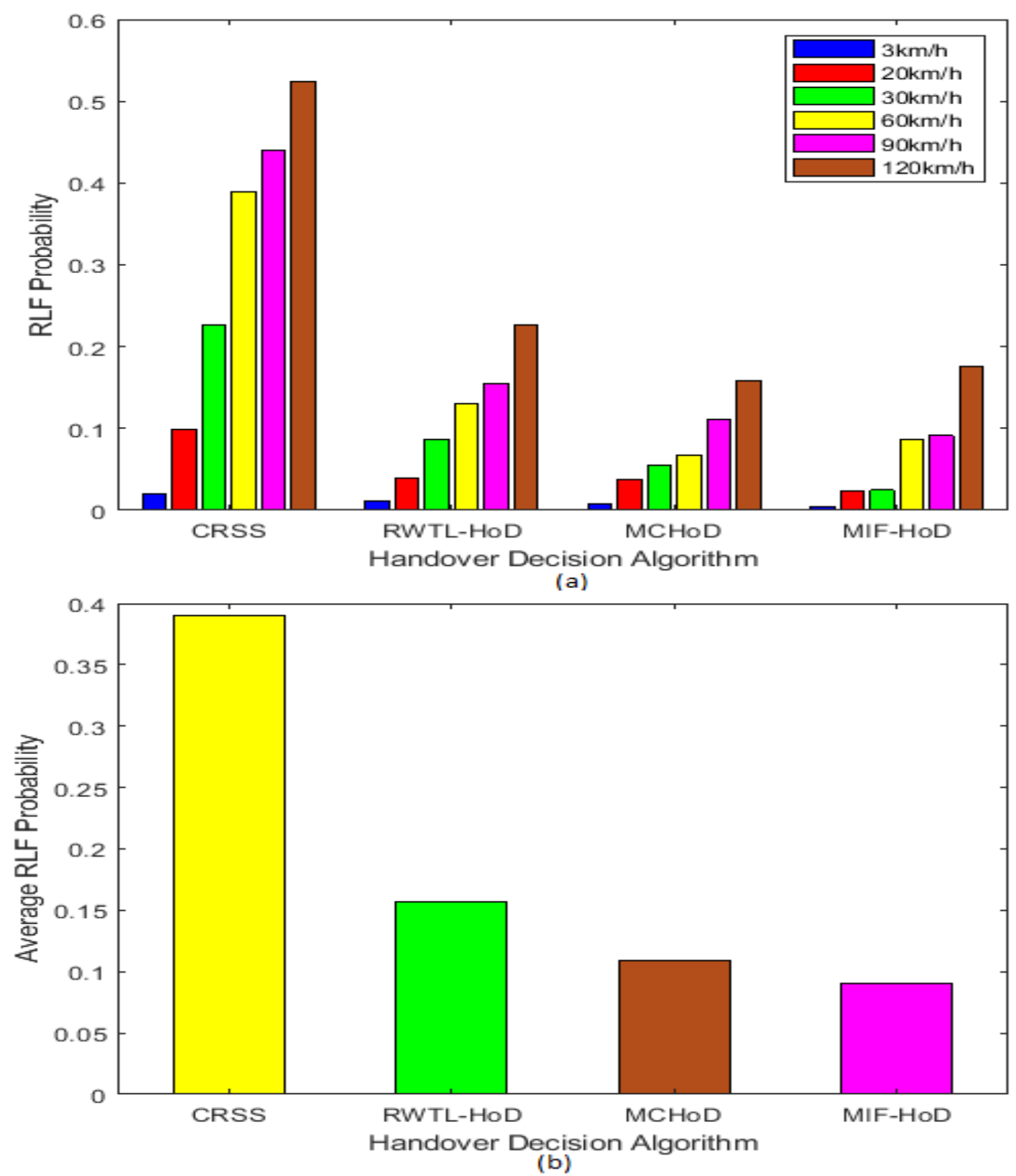

Figure 3: Probability of Radio Link Failure (RLF). (a) Failure at different UE's speeds and (b) average RLF for different decision algorithms

The plot of the Handover Ping Pong (HoPP) ratio against the speed is presented in Figure 4a. As expected, due to its reliance on only the downlink signal quality, the CRSS method shows the highest HoPP rate. Except for MCHoD which has insignificant ping-pong effects across the different speeds examined, other decision criteria show the extensive influence of speed over HoPP. Obviously, this phenomenon increases with increasing UE speed. In Figure 4a, as the RSS in the downlink tends to fluctuate due to shadow fading, cell selection becomes unstable, leading to a high HoPP ratio. The higher the HoPP, the more resources are wasted. In our algorithm, HoPP is minimized much better by using MCHoD where the handover is triggered if the requirements are met. Thus, the UE can be handed over to a suitable cell that satisfies the entire requirements for a successful handover to the target cell. The MCHoD has the best performance. 
International Journal of Computer Networks \& Communications (IJCNC) Vol.12, No.4, July 2020

It reduces the HoPP by approximately $81 \%$, 59\%, and 36\% over the CRSS, RWTL, and MIFHoD, respectively.

The resultant effect of HoF, RLF, and HoPP reflects on the Cumulative Distribution Function (CDF) probability UEs throughput. The higher the throughput value on the CDF probability plot, the better the performance of the system (Figure $4 \mathrm{~b}$ ). It can be observed, therefore, that the proposed MCHoD decision algorithm performs better than all the other algorithms evaluated in this work. In short, MCHoD is designed to select the best target cell based on multi-criteria decision method. This reduces the HoPP and increases the throughput gain.
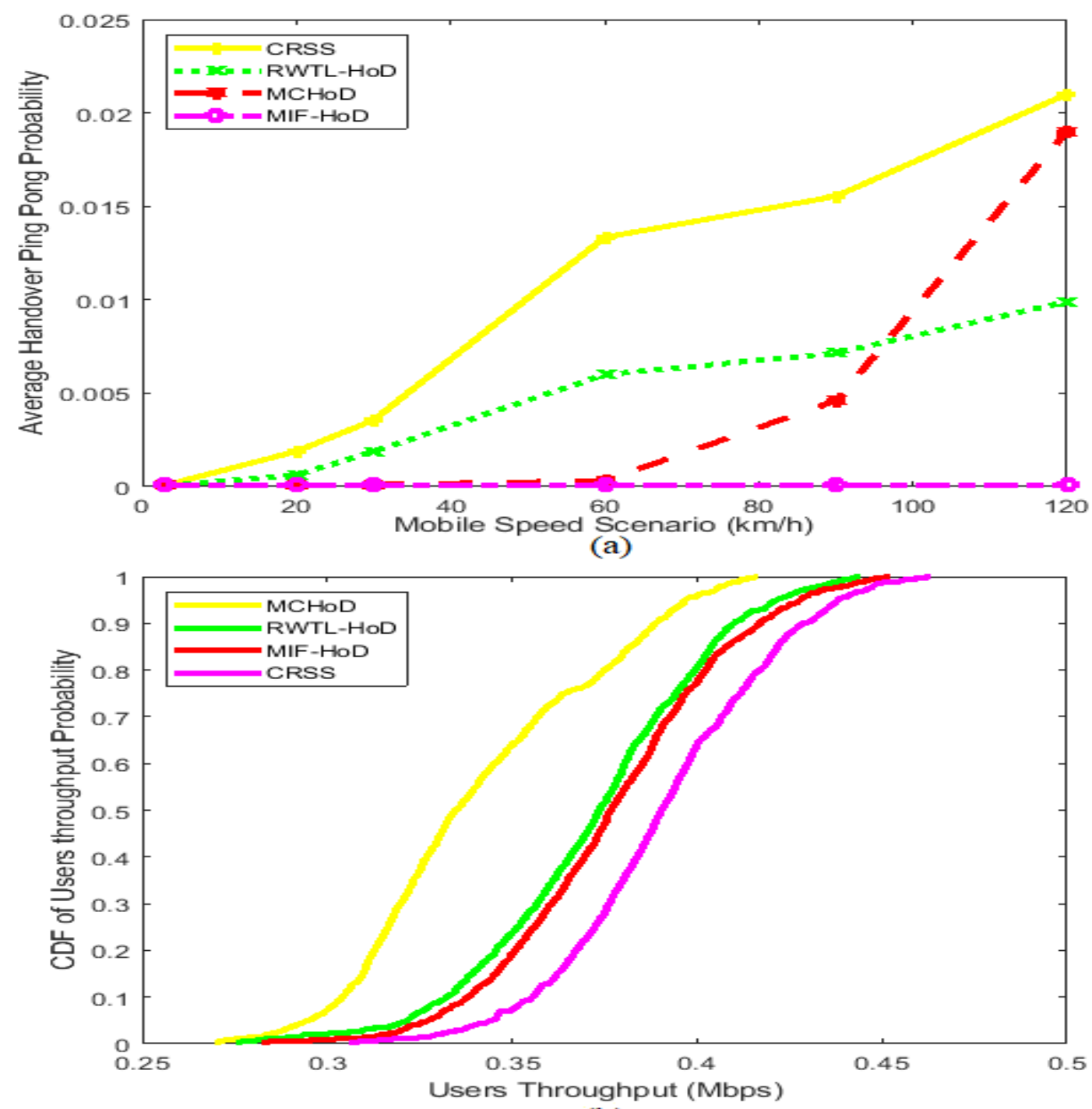

(b)

Figure 4: (a) Average Ping-Pong Handover probability over different speeds and (b) CDF of UEs throughputs

\section{Conclusion}

The proposed MCHoD performs better than previous handover decision algorithms as it demonstrate a significant reduction in HoF, RLF, and HoPP rates. This is an achievement and can 
International Journal of Computer Networks \& Communications (IJCNC) Vol.12, No.4, July 2020

be explained thus. First, there is a choice in the mapping of CCs, and PCC configured on MeNodeB for wider coverage, and the SCC on FeNodeB to extend the signal to the cell edge users. Second, the implementation of AMC schemes which allows UEs to be assigned the most suitable channel irrespective of variations in the channel conditions linearly increases the SINR which eventually leads to improvement in the entire system throughput. Third, the implementation of CRE techniques to resolve the load imbalance problem among the configured networks thereby offloading traffics from the congested MeNodeBs. One of the outstanding performances of the MCHoD is that it minimizes the effects of the parameters examined (RLF, $\mathrm{HoF}$, and HoPP) across the observed UE speeds. It also provides efficient resource utilization as a result of the increased number of aggregated CCs that can be deployed at an eNodeB and simultaneously assigned to a UE. This development is useful whenever there are a number of users that are required to add or remove one or more CCs. Finally, MCHoD contributes to the enhancement of the system performances by increasing user SINR, spectral efficiency, and reducing the user's outage probability. Since the future generation wireless communication will be built on these qualities, $\mathrm{MCHoD}$ stands a promising handover decision algorithm for carrier aggregation, Het-Nets deployment in LTE-A and future generation of wireless communication

As with every research, there are limitations and this study is no exception. Since carrier aggregation technique and heterogeneous networks deployment appears to be the prospect of wireless generation networks for higher capacity and data rate, further study needs to be conducted on effective deployment of Femtocell (FeNodeB) for high-speed outdoor application. Parameters like security, password protection, interoperability, etc. can also be included. Technically, a femtocell is deployed for use indoors, but in this study, it is considered for outdoor use and tested over different UE speeds. Certainly, its deployment outside is practicable but may result in a high rate of ping-pong especially with user speed above $120 \mathrm{~km} / \mathrm{h}$.

\section{CONFLICTS OF INTEREST}

The authors declare no conflict of interest.

\section{REFERENCES}

[1] S. Maaloul, M. Afif, and S. Tabbane, "Handover Decision in Heterogeneous Networks," in IEEE 30th International Conference on Advanced Information Networking and Applications, 2016, pp. 588-595.

[2] G. P. Pollini, “Trends in Handover Design,” IEEE Commun. Mag., vol. 34, no. 3, pp. 82-90, 1996.

[3] S. Dhar ROY, "Performance Evaluation of Signal Strength Based Handover Algorithms," Int'1 J. Commun. Netw. Syst. Sci., vol. 02, no. 07, pp. 657-663, 2009.

[4] I. Ken-Ichi, W. Soichi, S. Jen-Shew, and S. Takuro, "Performance of Handoff Algorithm Based on Distance and RSSI Measurements VOL. 51, NO. 6, NOVEMBER 2002,” IEEE Trans. Veh. Technol., vol. 51, no. 6, 2002.

[5] S. Lal and D. K. Panwar, "Coverage Analysis of Handoff Algorithm with Adaptive Hystersis Margin," in International conference on Information Technology, 2007, pp. 133-138.

[6] K. Yang, I. Gondal, B. Qiu, and L. S. Dooley, "Combined SINR Based Vertical Handoff Algorithm for Next Generation Heterogeneous Wireless Networks," in IEEE Globecom proceedings, 2007, pp. 4483-4487.

[7] A. K., N. K.; and a. D. P.Ayyappan, "SINR Based Vertical Handoff Scheme for QoS in Heterogeneous Wireless Networks," in International Conference on Future Computer and Communication (ICFCC 2009)., 2009, pp. 117-121.

[8] A. A. Bathich, M. D. Baba, R. A. Rahman, and U. T. Mara, "SINR Based Media Independent Handover in WiMAX and WLAN Networks," in international conference on Computier Application and iIdustrial Electronics (ICCAIE), 2011, pp. 331-334. 
International Journal of Computer Networks \& Communications (IJCNC) Vol.12, No.4, July 2020

[9] P. Baruah, N. Sarma, and S. S. Satapathy, "SINR based vertical handoff algorithm between GPRS and WiMAX networks," J. Theor. Appl. Inf. Technol., vol. 14, no. 2, pp. 107-110, 2010.

[10] H. Choi, “An Optimal Handover Decision for Throughput Enhancement," IEEE Commun. Lett., vol. 14, no. 9, pp. 851-853, 2010.

[11] G. Mahardhika, M. Ismail, and R. Nordin, "Multi-criteria vertical handover decision algorithm in heterogeneous wireless network," J. Theor. Appl. Inf. Technol., vol. 54, no. 2, pp. 339-345, 2015.

[12] M. A. Radhwan and A. Z. Zuriati, "Enhanced Handover Decision Algorithm in Heterogeneous Wireless network," Sensors, vol. 1626, no. 17, pp. 1-14, 2017.

[13] S. Pahal and P. Sehrawat, "Multi-Criteria Handoff Decision Algorithms In Wireless Networks," J. Mob. Comput. Appl., vol. 2, no. 2, pp. 2394-42, 2015.

[14] M. M. Alkhawlani, "Multi-Criteria Vertical Handover for Heterogonous Networks," Int. J. Wirel. Mob. Networks, vol. 3, no. 2, pp. 149-163, 2011.

[15] X. Lin, J. Andrews, and A. Ghosh, "Modeling, analysis and design for carrier aggregation in heterogeneous cellular networks," IEEE Transactions on Communications, pp. 1-12, 2012.

[16] X. Lin, J. G. Andrews, R. Ratasuk, B. Mondal, and A. Ghosh, "Carrier Aggregation in Heterogeneous Cellular Networks,” IEEE Wireless Communication Symposium, pp. 5199-5203, 2013.

[17] I. Shayea, M. Ismail, R. Nordin, and H. Mohamad, "Adaptive Handover Decision Algorithm Based on Multi-Influence Factors through Carrier Aggregation Implementation in LTE- Advanced System,” J. Comput. Networks Commun., vol. 2014, p. 8, 2014.

[18] 3GPP, "TR 136912 - V13.0.0 - LTE; Feasibility study for Further Advancements for E-UTRA (LTEAdvanced) (3GPP TR 36.912 version 13.0.0 Release 13)," 2016.

[19] 4G Americas, "LTE-Advanved: 'Carrier Aggregation Aggregation Technology Development and Deployment worldwide’ http://www.4gamericas.org/files/8414/1471/2230/,” 2014.

[20] T. Anti, "LTE-Advanced Standardization," in LTE-Advanced: 3GPP Solution for IMT-Advanced, 1st ed., H. Holma and A. Toskala, Eds. John Wiley \& Sons, 2012, pp. 8-13.

[21] L. Liu, M. Li, J. Zhou, X. She, L. Chen, Y. Sagae, and M. Iwamura, "Component Carrier Management for Carrier Aggregation in LTE-Advanced System," in IEEE 73RD Vehicular Technology Conference (VTC Spring), 2011, pp. 1-6.

[22] 3GPP, "3GPP TR 36.839 V11.0.0 (2012-09), Mobility enhancements in heterogeneous networks (Release 11)," 2012.

[23] K. Vasudeva, M. Simsek, D. Lopez-Perez and I. Guvenc and S. Meryem, "Analysis of Handover Failures in Heterogeneous Networks with Fading," IEEE Transactions on Vehicular Technology, vol. 66, no. 7, pp. 6060-6074, 2017.

[24] R. Kaur and H. Singh, "An Efficient Approach for Handover Decision Making In Wireless Networks,” Int. Res. J. Eng. Technol., vol. 05, no. 02, pp. 454-458, 2018.

[25] Sonia and G. S. Saini, "Optimized RSS based Algorithm for Heterogeneous Networks," Indian J. Sci. Technol., vol. 9, no. December, p. 6, 2016.

[26] P. Tillapart, T. Thumthawatworn, P. Viriyaphol, and P. Santiprabhob, "Intelligent Handover Decision Based on Fuzzy Logic for Heterogeneouse Wireless Networks," in Electrica/Electronics Engineering, Computer, Telecommunication and Information Technology (ECTI-CON), 2015, pp. 1-6.

[27] V. A. Narayanan, A. Rajeswari, and V. Sureshkumar, "AN Intelligent Vertical Handover Decision Algorithm for Heterogeneous Networks,” Am. J. Appl. Sci., vol. 11, no. 5, pp. 732-739, 2014.

[28] M. Khan, "Multi-criteria based vertical handover decision in heterogeneous wireless network," in Proceedings of the 30th Annual ACM Symposium on Applied Computing - SAC '15, 2015, vol. 2015, pp. 720-721.

[29] Y. D. Lee, S. D. Chun, S. J. Yi, S. J. Park, and S. H. Jung, "Carrier aggregation management method, system and devices., [Google Patents].," US 2011/0028148 A1, 2011.

[30] P. J. Song and J. Shin, "Method for handover in multi-carrier system, [Google Patents], US8688120 B2.," US8688120 B2., 2014.

[31] Z. Shen, A. Papasakellariou, G. Dirk, and F. Xu, "Overview of 3GPP LTE-Advanced Carrier Aggregation for 4G Wireless Communications,” IEEE Communications Magazine, no. February, pp. 122-130, 2012.

[32] L. Liu, M. Li, J. Zhou, X. She, L. Chen, Y. Sagae, and M. Iwamura, "Component Carrier Management for Carrier Aggregation in LTE-Advanced System," IEEE 69th Veh. Technol. Conf. spring (VTC2009-Spring), Barcelonal, Spain, pp. 1-6, 2011.

[33] "DOCOMO R4-102114: Simulation Assumption for mobility performance in Carrier Aggregation; 
DoCoMo, Montreal, Canada," 2010.

[34] 3GPP, "Radio Frequency (RF) system scenarios, (3GPP TR 36.942 version 14.0.0 Release 14), FRANCE," 2016.

[35] I. Mikio, K. Etemad, M. Fong, R. Nory, and R. Love, “Carrier Aggregation Framework in,” IEEE Commun. Mag., no. August, pp. 60-67, 2010.

[36] Qualcomm, "R1-100701 Importance of Serving Cell Association in Heterogeneous Networks," 2010, pp. 1-6.

[37] 3GPP, “TR 136 932 - V14.1.0 - LTE; Scenarios and requirements for small cell enhancements for EUTRA and E-UTRAN," 2017.

[38] 3GPP, "TS 136211 - V14.2.0, Physical channels and modulation, (3GPP TS 36.211 version 14.2.0 Release 14)," 2017.

[39] 3GPP, “TS 36.300 , Overall description ; 3GPP TS 36.300 version 13.9.0 (Release 13).," 2017.

\section{Authors}

Maryam Abdulazeez- Ahmed received her first degree in Electronics and Telecommunication Engineering from Kwara Polytechnic Ilorin Nigeria in 1989, Master of Engineering Management in 2011 from Universiti Putra Malaysia (UPM) and Ph.D. (in view), Wireless Communication Engineering from UPM. Her research interests include mobile cellular, wireless, and green communication. She has published several papers in journals and conferences.

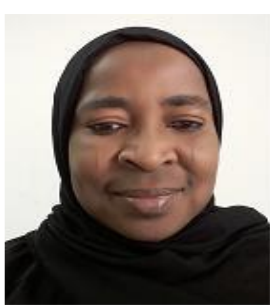

Nor Kamariah Noordin received her B.Sc. in Electrical Engineering from the University of Alabama, USA, in 1987. She became a tutor at the Department of Computer and Communication System Engineering, UPM, and obtained a Master Degree from Universiti Teknologi Malaysia (UTM) and Ph.D. from UPM. She is currently the Director of Corporate Planning Division at the Office of the ViceChancellor. She has received National, and research awards, being a researcher at the Wireless and Photonic Network Research Center of Excellence (WiPNET), UPM. She has over 100 publications in journals and conferences.

Aduwati Bint Sali is a Professor at Department of Computer and Communication Systems, Faculty of Engineering, UPM. She obtained her Ph.D. in Mobile and Satellite Communications from the University of Surrey in the United Kingdom, in July 2009, her MSc in Communications and Network Engineering from UPM, in April 2002, and her B. Eng in Electrical Electronics Engineering (Communications) from University of Edinburgh, UK, in 1999. Her research interests are radio resource management, MAC layer protocols, satellite communications, wireless sensor networks, satellite-assisted emergency

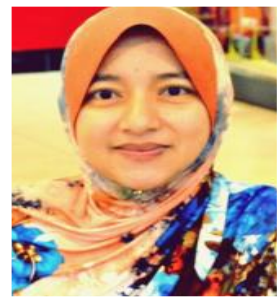
communications, and 3D video transmission over wireless networks. She is a researcher at the WiPNET, UPM.

Fazirulhisyam Hashim holds an MSc degree from Universiti Sains Malaysia and a Ph.D. in Telecommunications Engineering from the University of Sydney, Australia. His research interests include network security and quality of service of next-generation mobile networks, green communication systems, cognitive networks, and wireless sensor networks. He is a member of IEEE and ACM, and currently a senior lecturer and researcher at the Wireless and Photonic Network Research Center of Excellence (WiPNET), Universiti Putra Malaysia.

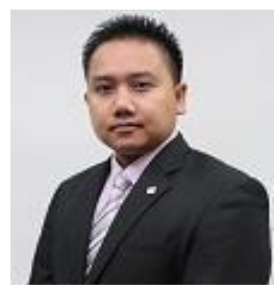

